\title{
How to Obtain Computational Completeness in P Systems with One Catalyst
}

\author{
Rudolf Freund \\ Technische Universität Wien, Institut für Computersprachen \\ Favoritenstr. 9, A-1040 Wien, Austria \\ rudi@emcc. at \\ Gheorghe Păun \\ Institute of Mathematics of the Romanian Academy \\ PO Box 1-764, 014700 Bucureşti, Romania, and \\ Department of Computer Science and Artificial Intelligence \\ University of Sevilla \\ Avda. Reina Mercedes s/n, 41012 Sevilla, Spain \\ gpaun@us.es, ghpaun@gmail.com
}

\begin{abstract}
Whether P systems with only one catalyst can already be computationally complete, is still an open problem. Here we establish computational completeness by using specific variants of additional control mechanisms. At each step using only multiset rewriting rules from one set of a finite number of sets of multiset rewriting rules allows for obtaining computational completeness with one catalyst and only one membrane. If the targets are used for choosing the multiset of rules to be applied, for getting computational completeness with only one catalyst more than one membrane is needed. If the available sets of rules change periodically with time, computational completeness can be obtained with one catalyst in one membrane. Moreover, we also improve existing computational completeness results for P systems with mobile catalysts and for P systems with membrane creation.
\end{abstract}

\section{Introduction}

P systems with catalytic rules were already considered in the originating papers for membrane systems, see [12]. In [4] two catalysts were shown to be sufficient for getting computational completeness (throughout this paper, with this notion we will indicate that all recursively enumerable sets of (vectors of) non-negative integers can be generated). Since then, it has become one of the most challenging open problems in the area of $\mathrm{P}$ systems, whether or not one catalyst might already be enough to obtain computational completeness.

Using additional control mechanisms as, for example, priorities or promoters/inhibitors, $\mathrm{P}$ systems with only one catalyst can be shown to be computationally complete, e.g., see Chapter 4 of [13]. On the other hand, additional features for the catalyst may be taken into account; for example, we may use bi-stable catalysts (catalysts switching between two different states) or mobile catalysts (catalysts able to cross membranes). Moreover, additional membrane features may be used, for example, membrane creation or controlling the membrane permeability by means of the operations $\delta$ and $\tau$.

$\mathrm{P}$ systems with mobile catalysts were introduced in [7], and their computational completeness was proved with using three membranes and targets of the forms here, out, and $i n_{j}$. We here improve this result by replacing the targets $i n_{j}$ with the weaker one $i n$.

$\mathrm{P}$ systems with membrane creation were introduced in [9], showing both their computational completeness and efficiency (the Hamiltonian path problem is solved in linear time in a semi-uniform way; 
this result was improved in [6], where a polynomial solution to the Subset Sum problem in a uniform way is provided). For proving computational completeness, in [9] (Theorem 2) P systems starting with one membrane, having four membranes at some time during the computation, using one catalyst, and also controlling the membrane permeability by means of the operations $\delta$ and $\tau$ are needed. However, as already shown in [11], P systems with one catalyst and using the operations $\delta$ and $\tau$ are computationally complete, hence, the membrane creation facility is not necessary for getting computational completeness in this framework. Here we improve the result shown in [9] from two points of view: (i) the control of membrane permeability is not used, and (ii) the maximal number of membranes used during a computation is two.

Recenty, several variants of $\mathrm{P}$ systems using only one catalyst together with control mechanisms for choosing the rules applicable in a computation step have been considered: for example, in [8] the rules are labeled with elements from an alphabet $H$ and in each step a maximal multiset of rules having the same label from $H$ is applied. In this paper, we will give a short proof for the computational completeness of these $P$ systems with label selection with only one catalyst in a single membrane. As a specific variant, for each membrane we can choose the rules according to the targets, and we will prove computational completeness for these $P$ systems with target selection with only one catalyst, but needing more than one membrane (such systems with only one membrane lead to the still open problem of catalytic $\mathrm{P}$ systems with one catalyst).

Regular control languages were considered already in [8] for the maximally parallel derivation mode, whereas in [1] computational completeness was proved for the sequential mode: there even only noncooperative rules were needed in one membrane for time-varying $\mathrm{P}$ systems to obtain computational completeness (in time-varying systems, the set of available rules varies periodically with time, i.e., the regular control language is of the very specific form $W=\left(U_{1} \ldots U_{p}\right)^{*}$, allowing to apply rules from a set $U_{i}$ in the computation step $p n+i, n \geq 0 ; p$ is called the period), but a bounded number of steps without applying any rule had to be allowed. We here prove that time-varying $P$ systems using the maximally parallel derivation mode in one membrane with only one catalyst are computationally complete with a period of six and the usual halting when no rule can be applied.

The new results exhibited in this paper first were presented in [5]. For the newest developments in the area of $\mathrm{P}$ systems we refer the reader to the $\mathrm{P}$ systems website [15].

\section{Prerequisites}

The set of non-negative integers is denoted by $\mathbb{N}$. An alphabet $V$ is a finite non-empty set of abstract symbols. Given $V$, the free monoid generated by $V$ under the operation of concatenation is denoted by $V^{*}$; the elements of $V^{*}$ are called strings, and the empty string is denoted by $\lambda ; V^{*} \backslash\{\lambda\}$ is denoted by $V^{+}$. Let $\left\{a_{1}, \cdots, a_{n}\right\}$ be an arbitrary alphabet; the number of occurrences of a symbol $a_{i}$ in a string $x$ is denoted by $|x|_{a_{i}}$. For a fixed sequence $\left\langle a_{1}, \cdots, a_{n}\right\rangle$ of the symbols in the alphabet $\left\{a_{1}, \cdots, a_{n}\right\}$, the Parikh vector associated with $x$ with respect to $\left\langle a_{1}, \cdots, a_{n}\right\rangle$ is $\left(|x|_{a_{1}}, \cdots,|x|_{a_{n}}\right)$; the Parikh image of a language $L$ over $\left\{a_{1}, \cdots, a_{n}\right\}$ is the set of all Parikh vectors of strings in $L$, and we denote it by $P s(L)$. For a family of languages $F L$, the family of Parikh images of languages in $F L$ is denoted by $P S F L$; for families of languages of a one-letter alphabet, the corresponding sets of non-negative integers are denoted by $N F L$.

A (finite) multiset over the (finite) alphabet $V, V=\left\{a_{1}, \cdots, a_{n}\right\}$, is a mapping $f: V \longrightarrow \mathbb{N}$ and represented by $\left\langle f\left(a_{1}\right), a_{1}\right\rangle \cdots\left\langle f\left(a_{n}\right), a_{n}\right\rangle$ or by any string $x$ the Parikh vector of which with respect to $\left\langle a_{1}, \cdots, a_{n}\right\rangle$ is $\left(f\left(a_{1}\right), \cdots, f\left(a_{n}\right)\right)$. In the following we will not distinguish between a vector $\left(m_{1}, \cdots, m_{n}\right)$, its representation by a multiset $\left\langle m_{1}, a_{1}\right\rangle \cdots\left\langle m_{n}, a_{n}\right\rangle$ or its representation by a string $x$ having 
the Parikh vector $\left(|x|_{a_{1}}, \cdots,|x|_{a_{n}}\right)=\left(m_{1}, \cdots, m_{n}\right)$. For a fixed sequence $\left\langle a_{1}, \cdots, a_{n}\right\rangle$ of the symbols in the alphabet $\left\{a_{1}, \cdots, a_{n}\right\}$, the representation of the multiset $\left\langle m_{1}, a_{1}\right\rangle \cdots\left\langle m_{n}, a_{n}\right\rangle$ by the string $a_{1}^{m_{1}} \cdots a_{n}^{m_{n}}$ is unique.

The family of regular and recursively enumerable string languages is denoted by $R E G$ and $R E$, respectively. For more details of formal language theory the reader is referred to the monographs and handbooks in this area as [2] and [14].

A register machine is a tuple $M=\left(m, B, l_{0}, l_{h}, P\right)$, where $m$ is the number of registers, $P$ is the set of instructions bijectively labeled by elements of $B, l_{0} \in B$ is the initial label, and $l_{h} \in B$ is the final label. The instructions of $M$ can be of the following forms:

- $l_{1}:\left(\operatorname{ADD}(j), l_{2}, l_{3}\right)$, with $l_{1} \in B \backslash\left\{l_{h}\right\}, l_{2}, l_{3} \in B, 1 \leq j \leq m$.

Increase the value of register $j$ by one, and non-deterministically jump to instruction $l_{2}$ or $l_{3}$. This instruction is usually called increment.

- $l_{1}:\left(\operatorname{SUB}(j), l_{2}, l_{3}\right)$, with $l_{1} \in B \backslash\left\{l_{h}\right\}, l_{2}, l_{3} \in B, 1 \leq j \leq m$.

If the value of register $j$ is zero then jump to instruction $l_{3}$, otherwise decrease the value of register $j$ by one and jump to instruction $l_{2}$. The two cases of this instruction are usually called zero-test and decrement, respectively.

- $l_{h}$ : HALT. Stop the execution of instructions of the register machine.

A configuration of a register machine is described by the contents of each register and by the value of the current label, which indicates the next instruction to be executed. Computations start by executing the first instruction of $P$ (labeled with $l_{0}$ ), and terminate with reaching the HALT instruction.

Register machines provide a simple computing model which is computationally complete (e.g., see [10]). For generating sets of vectors of non-negative integers, we start with empty registers, use the first two registers for the necessary computations and take as results the contents of the $k$ registers 3 to $k+2$ in all possible halting computations; during a computation of $M$, only the registers 1 and 2 can be decremented, and moreover, we assume the registers 1 and 2 to be empty at the end of a halting computation. In the following, we shall call a specific model of $\mathrm{P}$ systems computationally complete if and only if for any register machine $M$ we can effectively construct an equivalent $\mathrm{P}$ system $\Pi$ of that type simulating each step of $M$ in a bounded number of steps and yielding the same results.

\subsection{P Systems}

The basic ingredients of a (cell-like) $\mathrm{P}$ system are the membrane structure, the objects placed in the membrane regions, and the evolution rules. The membrane structure is a hierarchical arrangement of membranes. Each membrane defines a region/compartment, the space between the membrane and the immediately inner membranes; the outermost membrane is called the skin membrane, the region outside is the environment, also indicated by (the label) 0. Each membrane can be labeled, and the label (from a set $L a b$ ) will identify both the membrane and its region. The membrane structure can be represented by a rooted tree (with the label of a membrane in each node and the skin in the root), but also by an expression of correctly nested labeled parentheses. The objects (multisets) are placed in the compartments of the membrane structure and usually represented by strings, with the multiplicity of a symbol corresponding to the number of occurrences of that symbol in the string. The evolution rules are multiset rewriting rules of the form $u \rightarrow v$, where $u$ is a multiset of objects from a given set $O$ and $v=\left(b_{1}, \operatorname{tar}_{1}\right) \ldots\left(b_{k}, \operatorname{tar}_{k}\right)$ with $b_{i} \in O$ and tar $_{i} \in\{$ here, out, in $\}$ or tar $_{i} \in\{$ here, out $\} \cup\left\{\right.$ in $\left._{j} \mid j \in L a b\right\}, 1 \leq i \leq k$. Using such a rule means "consuming" the objects of $u$ and "producing" the objects $b_{1}, \ldots, b_{k}$ of $v$; the target indications 
(targets for short) here, out, and in mean that an object with the target here remains in the same region where the rule is applied, an object with the target out is sent out of the respective membrane (in this way, objects can also be sent to the environment, when the rule is applied in the skin region), while an object with the target in is sent to one of the immediately inner membranes, non-deterministically chosen, wheras with $i n_{j}$ this inner membrane can be specified directly. Usually, we omit the target here. With respect to the tree representation of the membrane structure of the P system, the target out means moving the object to the region represented by the parent node, and the target in means moving the object to a region represented by one of the children nodes; with the target $i n_{j}$ we can directly specify which of the children nodes is to be chosen.

Formally, a (cell-like) P system is a construct

$$
\Pi=\left(O, \mu, w_{1}, \ldots, w_{m}, R_{1}, \ldots, R_{m}, f\right)
$$

where $O$ is the alphabet of objects, $\mu$ is the membrane structure (with $m$ membranes), $w_{1}, \ldots, w_{m}$ are multisets of objects present in the $m$ regions of $\mu$ at the beginning of a computation $, R_{1}, \ldots, R_{m}$ are finite sets of evolution rules, associated with the regions of $\mu$, and $f$ is the label of the membrane region from which the outputs are taken ( $f=0$ indicates that the output is taken from the environment).

If a rule $u \rightarrow v$ has at least two objects in $u$, then it is called cooperative, otherwise it is called noncooperative. In catalytic $P$ systems we use non-cooperative as well as catalytic rules which are of the form $c a \rightarrow c v$, where $c$ is a special object - a so-called catalyst - which never evolves and never passes through a membrane (both these restrictions can be relaxed), but it just assists object $a$ to evolve to the multiset $v$. In a purely catalytic $P$ system we only allow catalytic rules. In both catalytic and purely catalytic P systems we replace $O$ by $O, C$ in order to specify those objects from $O$ which are the catalysts in the set $C$, i.e., we write

$$
\Pi=\left(O, C, \mu, w_{1}, \ldots, w_{m}, R_{1}, \ldots, R_{m}, f\right) .
$$

The evolution rules are used in the non-deterministic maximally parallel way, i.e., in any computation step of $\Pi$ we choose a multiset of rules from the sets $R_{1}, \ldots, R_{m}$ in such a way that no further rule can be added to it so that the obtained multiset would still be applicable to the existing objects in the membrane regions $1, \ldots, m$.

The membranes and the objects present in the compartments of a system at a given time form a configuration; starting from a given initial configuration and using the rules as explained above, we get transitions among configurations; a sequence of transitions forms a computation. A computation is halting if it reaches a configuration where no rule can be applied anymore. With a halting computation we associate a result, in the form of the number of objects present in membrane $f$ in the halting configuration. The set of non-negative integers and the set of (Parikh) vectors of non-negative integers obtained as results of halting computations in $\Pi$ are denoted by $N(\Pi)$ and $P S(\Pi)$, respectively.

The family of sets $Y(\Pi), Y \in\{N, P s\}$, computed by P systems with at most $m$ membranes and cooperative rules and with non-cooperative rules is denoted by $Y O P_{m}(c o o p)$ and $Y O P_{m}$ (ncoo), respectively. It is well known that for any $m \geq 1, Y R E G=Y O P_{m}(n c o o) \subset N O P_{m}(c o o p)=Y R E$, see [12].

The family of sets $Y(\Pi), Y \in\{N, P s\}$, computed by (purely) catalytic P systems with at most $m$ membranes and at most $k$ catalysts is denoted by $Y O P_{m}\left(\right.$ cat $\left._{k}\right)\left(Y O P_{m}\left(\right.\right.$ pcat $\left.\left._{k}\right)\right)$; from [4] we know that, with the results being sent to the environment, we have $Y O P_{1}\left(c_{2} t_{2}\right)=Y O P_{1}\left(\right.$ pcat $\left._{3}\right)=Y R E$.

If we allow catalysts to move from one membrane region to another one, then we speak of $P$ systems with mobile catalysts. The families of sets $N(\Pi)$ and $P s(\Pi)$ computed by $\mathrm{P}$ systems with at most $m$ membranes and $k$ mobile catalysts are denoted by $N_{m} P_{m}\left(\right.$ mcat $\left._{k}\right)$ and $P_{s O P}\left(\right.$ mcat $\left._{k}\right)$, respectively. 
For all the variants of $\mathrm{P}$ systems using rules of some type $X$ as defined above, we may consider systems containing only rules of the form $u \rightarrow v$ where $u \in O$ and $v=\left(b_{1}, \operatorname{tar}\right) \ldots\left(b_{k}\right.$, tar $)$ with $b_{i} \in O$ and tar $\in\{$ here,out, in $\}$ or tar $\in\{$ here, out $\} \cup\left\{\right.$ in $\left._{j} \mid j \in H\right\}, 1 \leq i \leq k$, i.e., in each rule there is only one target for all objects $b_{i}$; moreover, with the target in we assume all objects generated by the rules of the chosen multiset of rules applied to the objects in a specific region of the current configuration to choose the same inner membrane. If catalytic rules are considered, then we request the rules to be of the form $c a \rightarrow(c$, here $)\left(b_{1}\right.$, here $) \ldots\left(b_{k}\right.$,here $)$, as the catalyst is not allowed to move. P systems with target selection contain only these forms of rules; moreover, in each computation step, for each membrane region $i$ we choose a non-empty multiset (if it exists) of rules $R_{i}^{\prime}$ from $R_{i}$ having the same target $t a r$ - for different membranes these targets may be different - and apply $R_{i}^{\prime}$ in the maximally parallel way, i.e., the set $R_{i}^{\prime}$ cannot be extended by any further rule from $R_{i}$ with the target tar so that the obtained multiset of rules would still be applicable to the existing objects in the membrane region $i$. The family of sets $N(\Pi)$ and $P s(\Pi)$ computed by $\mathrm{P}$ systems with target selection with at most $m$ membranes and rules of type $X$ is denoted by $N O P_{m}(X, t s)$ and $P s O P_{m}(X, t s)$, respectively.

For all the variants of $\mathrm{P}$ systems of type $X$, we may consider to label all the rules in the sets $R_{1}, \ldots, R_{m}$ in a one-to-one manner by labels from a set $H$ and to take a set $W$ containing subsets of $H$. Then a $P$ system with label selection is a construct

$$
\Pi=\left(O, \mu, w_{1}, \ldots, w_{m}, R_{1}, \ldots, R_{m}, H, W, f\right)
$$

where $\Pi^{\prime}=\left(O, \mu, w_{1}, \ldots, w_{m}, R_{1}, \ldots, R_{m}, f\right)$ is a $\mathrm{P}$ system as defined above, $H$ is a set of labels for the rules in the sets $R_{1}, \ldots, R_{m}$, and $W \subseteq 2^{H}$. In any transition step in $\Pi$ we first select a set of labels $U \in W$ and then apply a non-empty multiset $R$ of rules such that all the labels of these rules in $R$ are in $U$ in the maximally parallel way, i.e., the set $R$ cannot be extended by any further rule with a label from $U$ so that the obtained multiset of rules would still be applicable to the existing objects in the membrane regions $1, \ldots, m$. The family of sets $N(\Pi)$ and $P s(\Pi)$ computed by $\mathrm{P}$ systems with label selection with at most $m$ membranes and rules of type $X$ is denoted by $N_{N O P}(X, l s)$ and ${ }_{s} O P_{m}(X, l s)$, respectively.

Another method to control the application of the labeled rules is to use control languages (see [8] and [1]). A controlled P system is a construct

$$
\Pi=\left(O, \mu, w_{1}, \ldots, w_{m}, R_{1}, \ldots, R_{m}, H, L, f\right)
$$

where $\Pi^{\prime}=\left(O, \mu, w_{1}, \ldots, w_{m}, R_{1}, \ldots, R_{m}, f\right)$ is a $\mathrm{P}$ system as defined above, $H$ is a set of labels for the rules in the sets $R_{1}, \ldots, R_{m}$, and $L$ is a string language over $2^{H}$ (each subset of $H$ represents an element of the alphabet for $L$ ) from a family $F L$. Every successful computation in $\Pi$ has to follow a control word $U_{1} \ldots U_{n} \in L$ : in transition step $i$, only rules with labels in $U_{i}$ are allowed to be applied (but again in the maximally parallel way, i.e., we have to apply a multiset $R$ of rules with labels in $U_{i}$ which cannot be extended by any rule with a label in $U_{i}$ such that the resulting multiset would still be applicable), and after the $n$-th transition, the computation halts; we may relax this end condition, i.e., we may stop after the $i$-th transition for any $i \leq n$, and then we speak of weakly controlled $P$ systems. If $L=\left(U_{1} \ldots U_{p}\right)^{*}$, $\Pi$ is called a (weakly) time-varying $P$ system: in the computation step $p n+i, n \geq 0$, rules from the set $U_{i}$ have to be applied; $p$ is called the period. The family of sets $Y(\Pi), Y \in\{N, P s\}$, computed by (weakly) controlled $\mathrm{P}$ systems and (weakly) time-varying $\mathrm{P}$ systems with period $p$, with at most $m$ membranes and rules of type $X$ as well as control languages in $F L$ is denoted by $Y O P_{m}(X, C(F L))\left(Y O P_{m}(X, w C(F L))\right)$ and $Y O P_{m}\left(X, T V_{p}\right)\left(Y O P_{m}\left(X, w T V_{p}\right)\right)$, respectively.

In the $P$ systems with membrane creation considered in this paper, besides the catalytic rules $c a \rightarrow$ $c(u, t a r)$ and the non-cooperative rules $a \rightarrow(u, t a r)$ we also use catalytic membrane creation rules of 
the form $c a \rightarrow c[u]_{i}$ (in the context of $c$, from the object $a$ a new membrane with label $i$ containing the multiset $u$ is generated) and membrane dissolution rules $a \rightarrow u \delta$ (we assume that no objects can be sent into a membrane which is going to be dissolved; with dissolving the membrane $i$ by applying $\delta$, all objects contained inside this membrane are collected in the region surrounding the dissolved membrane); in all cases, $c$ is a catalyst, $a$ is an object, $u$ is a multiset, and tar is a target of the form here, out, and $i n_{j}$. The family of sets $Y(\Pi), Y \in\{N, P s\}$, computed by such $\mathrm{P}$ systems with membrane creation and using at most $k$ catalysts, with $m$ initial membranes and having at most $h$ membranes during its computations is denoted by $Y P_{m, h}\left(c a t_{k}, m c r e\right)$.

\section{Computational Completeness of P Systems with Label Selection}

Theorem $1 Y O P_{1}\left(c a t_{1}, l s\right)=Y R E, Y \in\{N, P s\}$.

Proof. We only prove the inclusion $P s R E \subseteq P_{s} O P_{1}\left(c_{1} t_{1}, l s\right)$. Let us consider a register machine $M=\left(n+2, B, l_{0}, l_{h}, I\right)$ with only the first and the second register ever being decremented, and let $A=$ $\left\{a_{1}, \ldots, a_{n+2}\right\}$ be the set of objects for representing the contents of the registers 1 to $n+2$ of $M$. We construct the following P system:

$$
\begin{aligned}
\Pi & =\left(O,\{c\},[]_{1}, c d l_{0}, R_{1}, H, W, 0\right), \\
O & =A \cup B \cup\{c, d, \#\}, \\
H & =\left\{l, l^{\prime} \mid l \in B \backslash\left\{l_{h}\right\}\right\} \cup\left\{l_{\langle x\rangle} \mid x \in\left\{1,2,1^{\prime}, 2^{\prime}, d, \#\right\}\right\},
\end{aligned}
$$

and the rules for $R_{1}$ and the sets of labels in $W$ are defined as follows:

A. Let $l_{i}:\left(\operatorname{ADD}(r), l_{j}, l_{k}\right)$ be an ADD instruction in $I$. If $r>2$, then the (labeled) rules

$$
l_{i}: l_{i} \rightarrow l_{j}\left(a_{r}, \text { out }\right), l_{i}^{\prime}: l_{i} \rightarrow l_{k}\left(a_{r}, \text { out }\right),
$$

are used, and for $r \in\{1,2\}$, we take the rules

$$
l_{i}: l_{i} \rightarrow l_{j} a_{r}, l_{i}^{\prime}: l_{i} \rightarrow l_{k} a_{r} .
$$

In both cases, we define $\left\{l_{i}, l_{i}^{\prime}\right\}$ to be the corresponding set of labels in $W$. The contents of each register $r, r \in\{1,2\}$, is represented by the number of objects $a_{r}$ present in the skin membrane; any object $a_{r}$ with $r \geq 3$ is immediately sent out into the environment.

B. The simulation of a SUB instruction $l_{i}:\left(\operatorname{SUB}(r), l_{j}, l_{k}\right)$, for $r \in\{1,2\}$, is carried out by the following rules and the corresponding sets of labels in $W$ :

For the case that the register $r, r \in\{1,2\}$, is not empty we take the (labeled) rules

$$
l_{i}: l_{i} \rightarrow l_{j}, l_{\langle r\rangle}: c a_{r} \rightarrow c, l_{\langle d\rangle}: c d \rightarrow c \#,
$$

(if no symbol $a_{r}$ is present, i.e., if the register $r$ is empty, then the trap symbol \# is introduced by the rule $\left.l_{\langle d\rangle}: c d \rightarrow c \#\right)$.

For the case that the register $r$ is empty, we take the (labeled) rules

$$
l_{i}^{\prime}: l_{i} \rightarrow l_{k}, l_{\left\langle r^{\prime}\right\rangle}: c a_{r} \rightarrow c \#
$$

(if at least one symbol $a_{r}$ is present, i.e., if the register $r$ is not empty, then the trap symbol \# is introduced by the rule $\left.l_{\left\langle r^{\prime}\right\rangle}: c a_{r} \rightarrow c \#\right)$. 
The corresponding sets of labels to be taken into $W$ are $\left\{l_{i}, l_{\langle r\rangle}, l_{\langle d\rangle}\right\}$ and $\left\{l_{i}^{\prime}, l_{\left\langle r^{\prime}\right\rangle}\right\}$, respectively. In both cases, the simulation of the SUB instruction works correctly if we have made the right choice.

C. We also add the labeled rule $l_{\langle \#\rangle}: \# \rightarrow \#$ to $R_{1}$ and the set $\left\{l_{\langle \#\rangle}\right\}$ to $W$, hence, the computation cannot halt once the trap symbol \# has been generated.

In sum, we observe that each computation step in $M$ is simulated by exactly one computation step in $\Pi$; moreover, such a simulating computation in $\Pi$ halts if and only if the corresponding computation in $M$ halts (as soon as the label $l_{h}$ appears, no rule can be applied anymore in $\Pi$, as we have not defined any rule for the HALT instruction of $M$ ). If at some moment we make the wrong choice when trying to simulate a SUB instruction and have to generate the trap symbol \#, the computation will never halt. Hence, we have shown $P s(M)=P s(\Pi)$, which completes the proof.

\section{Computational Completeness of P Systems with Target Selection}

Theorem $2 Y_{Y O P}\left(c a t_{1}, t s\right)=Y R E, Y \in\{N, P s\}$.

Proof. We only prove the inclusion $P s R E \subseteq P_{s} O_{7}\left(c a t_{1}, t s\right)$. Let us consider a register machine $M=\left(n+2, B, l_{0}, l_{h}, I\right)$ with only the first and the second register ever being decremented, and let $A=$ $\left\{a_{1}, \ldots, a_{n+2}\right\}$ be the set of objects for representing the contents of the registers 1 to $n+2$ of $M$. The set of labels $B \backslash\left\{l_{h}\right\}$ is divided into three disjoint subsets:

$$
\begin{aligned}
B_{+} & =\left\{l \mid l_{i}:\left(\operatorname{ADD}(r), l_{j}, l_{k}\right) \in I\right\}, \\
B_{-r} & =\left\{l \mid l_{i}:\left(\operatorname{SUB}(r), l_{j}, l_{k}\right) \in I\right\}, r \in\{1,2\}
\end{aligned}
$$

moreover, we define

$$
\begin{aligned}
B_{-} & =B_{-1} \cup B_{-2}, \\
B_{-}^{\prime} & =\left\{l^{\prime} \mid l \in B_{-}\right\}, \\
B_{-}^{\prime \prime} & =\left\{l^{\prime \prime} \mid l \in B_{-}\right\}, \text {and } \\
B^{\prime} & =B_{+} \cup B_{-} \cup B_{-}^{\prime} \cup B_{-}^{\prime \prime} .
\end{aligned}
$$

We construct the following P system:

$$
\begin{aligned}
\Pi & =\left(O,\{c\},\left[[]_{2} \ldots[]_{7}\right]_{1}, w_{1}, \ldots, w_{7}, R_{1}, \ldots, R_{7}, 0\right) \\
O & =A \cup B^{\prime} \cup\left\{a_{1}^{\prime}, a_{2}^{\prime}, c, d, \#\right\}
\end{aligned}
$$

with $w_{1}=l_{0}, w_{2}=c$, and $w_{i}=\lambda$ for $3 \leq i \leq 7$. In order to make argumentation easier, in the following we refer to the membrane labels 1 to 7 according to the following table:

\begin{tabular}{|l|l|l|l|l|l|l|}
\hline 1 & 2 & 3 & 4 & 5 & 6 & 7 \\
\hline skin & - & $0_{1}$ & $0_{2}$ & -1 & -2 & + \\
\hline
\end{tabular}

The sets of rules now are constructed as follows:

A. The simulation of any instruction from $I$ starts in the skin membrane with moving all objects except the output symbols $a_{r}, 3 \leq r \leq n+2$, into an inner membrane; according to the definition, taking the target in means choosing one of the inner membranes in a non-deterministic way, but the same 
membrane for all objects to be moved in. The output symbols $a_{r}, 3 \leq r \leq n+2$, are sent out into the environment by $a_{r} \rightarrow\left(a_{r}\right.$, out $)$, thus yielding the result of a halting computation as the number of symbols $a_{r}$ sent out into the environment during this computation. In case some copies of the output symbols $a_{r}$, $3 \leq r \leq n+2$, are present in the skin membrane, at any time we may either select the target out to send all these objects out into the environment or else select the target in in order to start the simulation of the next instruction. Choosing the target out or in always is done in a non-deterministic way. Hence, in sum we get

$$
\begin{aligned}
R_{1} & =\left\{x \rightarrow(x, \text { in }) \mid x \in B_{+} \cup B_{-} \cup\left\{a_{1}, a_{2}, a_{1}^{\prime}, a_{2}^{\prime}, \#\right\}\right\} \cup\left\{x \rightarrow(x d, \text { in }) \mid x \in B_{-}^{\prime}\right\} \\
& \cup\left\{a_{r} \rightarrow\left(a_{r}, \text { out }\right) \mid 3 \leq r \leq n+2\right\} .
\end{aligned}
$$

B. For the simulation of an ADD instruction $l_{i}:\left(\operatorname{ADD}(r), l_{j}, l_{k}\right) \in I$ all non-terminal symbols (all symbols except $a_{r}, r \geq 3$ ) are expected to have been sent to membrane + :

$$
\begin{aligned}
R_{+} & =\left\{l_{i} \rightarrow\left(l_{j} a_{r}, \text { out }\right), l_{i} \rightarrow\left(l_{k} a_{r}, \text { out }\right) \mid l_{i}:\left(\operatorname{ADD}(r), l_{j}, l_{k}\right) \in I\right\} \\
& \cup\left\{l \rightarrow(\#, \text { out }) \mid l \in B^{\prime} \backslash B_{+}\right\} \\
& \cup\left\{x \rightarrow(x, \text { out }) \mid x \in\left\{a_{1}, a_{2}, \#\right\}\right\} .
\end{aligned}
$$

If the symbols arrive in membrane + with a label $l \in B^{\prime} \backslash B_{+}$, then the trap symbol \# is generated and the computation will never halt.

C. The simulation of a SUB instruction $l_{i}:\left(\operatorname{SUB}(r), l_{j}, l_{k}\right)$ is carried out in two steps for the zero test, i.e., when the register $r$ is empty, using (the rules in) membrane $0_{r}$ and in five steps for decrementing the number of symbols $a_{r}$, first using membrane $-_{r}$ to mark the corresponding symbols $a_{r}$ into $a_{r}^{\prime}$ and then using the catalyst $c$ in membrane - to erase one of these primed objects; the marking procedure is necessary to guarantee that the catalyst erases the right object. For $r \in\{1,2\}$, we define the following sets of rules:

$$
\begin{aligned}
R_{0_{r}}= & \left\{l_{i} \rightarrow\left(l_{k}, \text { out }\right), a_{r} \rightarrow(\#, \text { out }) \mid l_{i}:\left(\operatorname{SUB}(r), l_{j}, l_{k}\right) \in I\right\} \\
\cup & \left\{l \rightarrow(\#, \text { out }) \mid l \in B^{\prime} \backslash B_{-r}\right\} \\
& \cup\left\{x \rightarrow(x, \text { out }) \mid x \in\left\{a_{3-r}, \#\right\}\right\} .
\end{aligned}
$$

If the number of objects $a_{r}$ is not zero, i.e., if the register $r$ is not empty, the introduction of the trap symbol \# causes the computation to never halt. On the other hand, if we want to decrement the register, we have to guarantee that exactly one symbol $a_{r}$ is erased:

$$
\begin{aligned}
R_{-r} & =\left\{l_{i} \rightarrow\left(l_{i}^{\prime}, \text { out }\right) \mid l_{i} \in B_{-r}\right\} \cup\left\{a_{r} \rightarrow\left(a_{r}^{\prime}, \text { out }\right)\right\} \\
& \cup\left\{l \rightarrow(\#, \text { out }) \mid l \in B^{\prime} \backslash B_{-r}\right\} \\
& \cup\left\{x \rightarrow(x, \text { out }) \mid x \in\left\{a_{3-r}, \#\right\}\right\} .
\end{aligned}
$$

The whole multiset of objects via the skin membrane now has to enter membrane -; here the dummy symbol $d$ guarantees that the catalyst cannot do nothing if no primed symbol $a_{r}^{\prime}$ has arrived; again the generation of \# causes the computation to not halt anymore:

$$
\begin{aligned}
R_{-} & =\left\{l_{i}^{\prime} \rightarrow l_{j}^{\prime \prime}, \text { ca } \rightarrow c, l_{i}^{\prime \prime} \rightarrow \#, l_{i}^{\prime \prime} \rightarrow\left(l_{j}, \text { out }\right) \mid l_{i}:\left(\operatorname{SUB}(r), l_{j}, l_{k}\right) \in I\right\} \\
& \cup\{c d \rightarrow c \#, d \rightarrow(\lambda, \text { out })\} \cup\left\{a_{r}^{\prime} \rightarrow\left(a_{r}, \text { out }\right) \mid r \in\{1,2\}\right\}, \\
& \cup\left\{l \rightarrow(\#, \text { out }) \mid l \in B^{\prime} \backslash B_{-}^{\prime \prime}\right\} \\
& \cup\left\{x \rightarrow(x, \text { out }) \mid x \in\left\{a_{3-r}, \#\right\}\right\} .
\end{aligned}
$$

The end of the simulation of the SUB instruction $l_{i}:\left(\operatorname{SUB}(r), l_{j}, l_{k}\right)$ in membrane - takes two steps: first,we apply $l_{i}^{\prime} \rightarrow l_{j}^{\prime \prime}$ and $c a_{r}^{\prime} \rightarrow c$, thus erasing exactly one symbol $a_{r}^{\prime}$, which corresponds to decrement 
register $r$; in the second step, we send out the label $l_{j}$ by using $l_{i}^{\prime \prime} \rightarrow\left(l_{j}\right.$, out $)$ together with the remaining symbols $a_{r}$ by using $a_{r}^{\prime} \rightarrow\left(a_{r}\right.$, out $)$ and all symbols $a_{3-r}$ by using $a_{3-r} \rightarrow\left(a_{3-r}\right.$, out $)$. The additional symbol $d$ generated in the first step in the skin membrane is eliminated by applying the rule $d \rightarrow(\lambda$, out $)$. These two steps cannot be interchanged, as with using the target out first we would have to use the rule $l_{i}^{\prime} \rightarrow(\#$, out $)$, thus introducing the trap symbol \#.

If in any of the membranes $R_{0_{r}}, R_{-_{r}}, r \in\{1,2\}$, and $R_{-}$the symbols arrive with the wrong label $l \in B^{\prime}$, then the trap symbol \# is generated and the computation will never halt.

We finally observe that a computation in $\Pi$ halts if and only if the final label $l_{h}$ appears (and then stays in the skin membrane) and no trap symbol \# is present, hence, we conclude $\operatorname{Ps}(M)=P s(\Pi)$.

To eventually reduce the number of inner membranes remains as a challenging task for future research.

\section{Computational Completeness of Time-Varying P Systems}

Theorem $3 Y O P_{1}\left(c_{1}, \alpha T V_{6}\right)=Y R E, \alpha \in\{\lambda, w\}, Y \in\{N, P s\}$.

Proof. We only prove the inclusion $P_{S R E} \subseteq P_{S} O P_{1}\left(c_{1} t_{1}, T V_{6}\right)$. Let us consider a register machine $M=\left(n+2, B, l_{0}, l_{h}, I\right)$ with only the first and the second register ever being decremented. Again, we define $A=\left\{a_{1}, \ldots, a_{n+2}\right\}$ and divide the set of labels $B \backslash\left\{l_{h}\right\}$ into three disjoint subsets:

$$
\begin{aligned}
B_{+} & =\left\{l_{i} \mid l_{i}:\left(\operatorname{ADD}(r), l_{j}, l_{k}\right) \in I\right\}, \\
B_{-r} & =\left\{l_{i} \mid l_{i}:\left(\operatorname{SUB}(r), l_{j}, l_{k}\right) \in I\right\}, r \in\{1,2\} ;
\end{aligned}
$$

moreover, we define $B_{-}=B_{-1} \cup B_{-2}$ as well as

$$
B^{\prime}=\left\{l, \tilde{l}, \hat{l} \mid l \in B \backslash\left\{l_{h}\right\}\right\} \cup\left\{l^{-}, l^{0}, \bar{l}^{-}, \bar{l}^{0}, \mid l \in B_{-}\right\} .
$$

The main challenge in the construction for the time-varying P system $\Pi$ is that the catalyst has to fulfill its task to erase an object $a_{r}, r \in\{1,2\}$, for both objects in the same membrane where all other computations are carried out, too; hence, at a specific moment in the cycle of period six, parts of simulations of different instructions have to be coordinated in parallel. The basic components of the time-varying $\mathrm{P}$ system $\Pi$ are defined as follows (we here do not distinguish between a rule and its label):

$$
\begin{aligned}
\Pi & =\left(O,\{c\},[]_{1}, c l_{0}, R_{1} \cup \cdots \cup R_{6}, R_{1} \cup \cdots \cup R_{6},\left(R_{1} \ldots R_{6}\right)^{*}, 0\right), \\
O & =A \cup\left\{a_{1}^{\prime}, a_{2}^{\prime}\right\} \cup B^{\prime} \cup\left\{c, h, l_{h}, \#\right\} .
\end{aligned}
$$

We now list the rules in the sets of rules $R_{i}$ to be applied in computation steps $6 n+i, n \geq 0,1 \leq i \leq 6$ :

$\mathbf{R}_{1}$ : in this first step of the cycle, especially all the ADD instructions are simulated, i.e., for each $l_{i}:\left(\operatorname{ADD}(r), l_{j}, l_{k}\right) \in I$ we take

$c l_{i} \rightarrow c a_{r} \tilde{l}_{j}, c l_{i} \rightarrow c a_{r} \tilde{l}_{k}$ for $r \in\{1,2\}$ as well as $c l_{i} \rightarrow c\left(a_{r}\right.$, out $) \tilde{l}_{j}, c l_{i} \rightarrow c\left(a_{r}\right.$, out $) \tilde{l}_{k}$ for $3 \leq r \leq n+2$ (in order to obtain the output in the environment, for $r \geq 3$ we have to take $\left(a_{r}\right.$, out $)$ instead of $\left.a_{r}\right)$; only in the sixth step of the cycle, from $\tilde{l}_{j}$ and $\tilde{l}_{k}$ the corresponding unmarked labels $l_{j}$ and $l_{k}$ will be generated;

$c l \rightarrow \mathrm{cl}^{-}, \mathrm{cl} \rightarrow \mathrm{cl}^{0}$ initiate the simulation of a SUB instruction for register 1 labeled by $l \in B_{-1}$, i.e., we make a non-deterministic guess whether register $r$ is empty (with introducing $l^{0}$ ) or not (with introducing $l^{-}$); 
$c l \rightarrow c \hat{l}$ marks a label $l \in B_{-2}$ (the simulation of such a SUB instruction for register 2 will start in step 4 of the cycle);

$\# \rightarrow$ \# keeps the trap symbol \# alive guaranteeing an infinite loop once \# has been generated;

$h \rightarrow \lambda$ eliminates the auxiliary object $h$ which eventually has been generated two steps before ( $h$ is needed for simulating the decrement case of SUB instructions).

$\mathbf{R}_{2}$ : in the second and the third step, the SUB instructions on register 1 are simulated, i.e., for all $l \in B_{-1}$ we start with

$c a_{1} \rightarrow c a_{1}^{\prime}$ (if present, exactly one copy of $a_{1}$ can be primed, but only if a label $l^{-}$for some $l$ from $B_{-1}$ is present) and

$l^{-} \rightarrow \bar{l}^{-} h, l^{0} \rightarrow \bar{l}^{0}$ for all $l \in B_{-1}$;

all other labels $\tilde{l}$ for $l \in B$ block the catalyst $c$ from erasing a copy of $a_{1}$ by forcing the application of the corresponding rules $c \tilde{l} \rightarrow c \tilde{l}$ for $c$ in order to avoid the introduction of the trap symbol \# by the enforced application of a rule $\tilde{l} \rightarrow \#$, i.e., we take

$c \tilde{l} \rightarrow c \tilde{l}, \tilde{l} \rightarrow \#$ for all $l \in B$, and

$c \hat{l} \rightarrow c \hat{l}, \hat{l} \rightarrow \#$ for all $l \in B_{-2}$;

$\# \rightarrow$ \# keeps the computation alive once the trap symbol has been introduced.

$\mathbf{R}_{3}$ : for all $l_{i}:\left(\operatorname{SUB}(1), l_{j}, l_{k}\right) \in I$ we take

$c \vec{l}_{i}^{0} \rightarrow c \tilde{l}_{k}, a_{1}^{\prime} \rightarrow \#, \vec{l}_{i}^{0} \rightarrow \#$ (zero test; if a primed copy of $a_{1}$ is present, then the trap symbol \# is generated);

$\bar{l}_{i}^{-} \rightarrow \tilde{l}_{j}, c a_{1}^{\prime} \rightarrow c, c h \rightarrow c \#$ (decrement; the auxiliary symbol $h$ is needed to keep the catalyst $c$ busy with generating the trap symbol \# if we have taken the wrong guess when assuming the register 1 to be non-empty);

$c \tilde{l} \rightarrow c \tilde{l}, \tilde{l} \rightarrow \#$ for all $l \in B$ (with these labels, we just pass through this step);

$c \hat{l} \rightarrow c \hat{l}, \hat{l} \rightarrow \#$ for all $l \in B_{-2}$ (these labels pass through this step to become active in the next step);

$\# \rightarrow$ \#.

$\mathbf{R}_{4}$ : in the fourth step, the simulation of SUB instructions on register 2 is initiated by using

$c \hat{l} \rightarrow c l^{-}, c \hat{l} \rightarrow c l^{0}$ for all $l \in B_{-2}$, i.e., we make a non-deterministic guess whether register $r$ is empty (with introducing $l^{0}$ ) or not (with introducing $l^{-}$);

$c \tilde{l} \rightarrow c \tilde{l}, \tilde{l} \rightarrow \#$ for all $l \in B$ (with all other labels, we only pass through this step);

$\# \rightarrow \#$,

$h \rightarrow \lambda$ (if $h$ has been introduced by $l^{-} \rightarrow \bar{l}^{-} h$ in the second step for some $l \in B_{-1}$, we now erase it).

$\mathbf{R}_{5}$ : in the fifth and the sixth step, the SUB instructions on register 2 are simulated, i.e., for all $l \in B_{-2}$ we start with

$c a_{2} \rightarrow c a_{2}^{\prime}$ (if present, exactly one copy of $a_{2}$ can be primed) and

$l^{-} \rightarrow \bar{l}^{-} h, l^{0} \rightarrow \bar{l}^{0}$ for all $l \in B_{-2}$;

$c_{1} \tilde{l} \rightarrow c_{1} \tilde{l}, \tilde{l} \rightarrow \#$ for all $l \in B$

$\# \rightarrow$ \#.

$\mathbf{R}_{6}$ : the simulation of SUB instructions $l_{i}:\left(\operatorname{SUB}(2), l_{j}, l_{k}\right) \in I$ on register 2 is finished by

$c \bar{l}_{i}^{0} \rightarrow c l_{k}, a_{2}^{\prime} \rightarrow \#, \bar{l}_{i}^{0} \rightarrow \#$ (zero test; if a primed copy of $a_{2}$ is present, then the trap symbol \# is generated);

$\bar{l}_{i}^{-} \rightarrow l_{j}, c a_{2}^{\prime} \rightarrow c, c h \rightarrow c \#$ (decrement; the auxiliary symbol $h$ is needed to keep the catalyst $c$ busy with generating the trap symbol \# if we have taken the wrong guess when assuming the register 2 to be non-empty; if it is not used, it can be erased in the next step by using $h \rightarrow \lambda$ in $R_{1}$ );

$c \tilde{l} \rightarrow c l, \tilde{l} \rightarrow \#$ for all $l \in B$ 
$\# \rightarrow \#$.

Without loss of generality, we may assume that the final label $l_{h}$ in $M$ is only reached by using a zero test on register 2; then, at the beginning of a new cycle, after a correct simulation of a computation from $M$ in the time-varying $\mathrm{P}$ system $\Pi$ no rule will be applicable in $R_{1}$ (another possibility would be to take $c \bar{l}_{i}^{0} \rightarrow c$ instead of $c l_{i}^{0} \rightarrow c l_{h}$ in $R_{6}$ ).

At the end of the cycle, in case all guesses have been correct, the requested instruction of $M$ has been simulated and the label of the next instruction to be simulated is present in the skin membrane. Only in the case that $M$ has reached the final label $l_{h}$, the computation in $\Pi$ halts, too, but only if during the simulation of the computation of $M$ in $\Pi$ no trap symbol \# has been generated; hence, we conclude $P s(M)=P s(\Pi)$.

\section{Computational Completeness of P Systems with Membrane Creation}

Theorem $4 Y_{Y O P_{1,2}}\left(\right.$ cat $_{1}$, mcre $)=Y R E, Y \in\{N, P s\}$.

Proof. We only prove the inclusion $P S R E \subseteq P_{S} O P_{1,2}\left(\right.$ cat $_{1}$, mcre $)$. Let us consider a register machine $M=\left(n+2, B, l_{0}, l_{h}, I\right)$ with only the first and the second register ever being decremented. Again we define $A=\left\{a_{1}, \ldots, a_{n+2}\right\}$ as the set of objects for representing the contents of the registers 1 to $n+2$ of $M$. We construct the following P system:

$$
\begin{aligned}
& \Pi=\left(O,\{c\},[]_{1}, c d l_{0}, R_{1}, R_{2}, R_{3}, 0\right), \\
& O=A \cup\left\{l, l^{\prime}, l^{\prime \prime} \mid l \in B\right\} \cup\left\{c, d, d^{\prime}, d^{\prime \prime}\right\}
\end{aligned}
$$

and the sets of rules are constructed as follows.

A. For each ADD instruction $l_{i}:\left(\operatorname{ADD}(r), l_{j}, l_{k}\right)$ in $I$, the rules

$$
\begin{array}{ll}
\text { step 1: } & l_{i} \rightarrow l_{i}^{\prime}, d \rightarrow d^{\prime}, \\
\text { step 2: } & l_{i}^{\prime} \rightarrow a_{r} l_{j}, l_{i}^{\prime} \rightarrow a_{r} l_{k}, d^{\prime} \rightarrow d,
\end{array}
$$

are taken into $R_{1}$ and obviously simulate an ADD instruction in two steps. We also add the rules $a_{r} \rightarrow$ $\left(a_{r}\right.$, out $)$ for $3 \leq r \leq n+2$ to $R_{1}$; thus, in any moment, every copy of $a_{r}, 3 \leq r \leq n+2$, present in the skin membrane is sent out to the environment.

B. For each SUB instruction $l_{i}:\left(\operatorname{SUB}(r), l_{j}, l_{k}\right)$ in $I$, the following rules in $R_{1}$ and $R_{r+1}, r \in\{1,2\}$, are used:

\begin{tabular}{c|c|c} 
Step & $R_{1}$ & $R_{r+1}$ \\
\hline 1 & $c l_{i} \rightarrow c\left[l_{i}\right]_{r+1}, d \rightarrow d^{\prime}$ & - \\
2 & $c a_{r} \rightarrow c\left(a_{r}, i n_{r+1}\right), d^{\prime} \rightarrow\left(d^{\prime}, i n_{r+1}\right)$ & $l_{i} \rightarrow l_{i}^{\prime}$ \\
3 & - & $a_{r} \rightarrow \lambda \delta, l_{i}^{\prime} \rightarrow l_{i}^{\prime \prime}, d^{\prime} \rightarrow d^{\prime \prime}$ \\
4 & $c l_{i}^{\prime \prime} \rightarrow c l_{j}, d^{\prime \prime} \rightarrow d$ & $l_{i}^{\prime \prime} \rightarrow l_{k}, d^{\prime \prime} \rightarrow d \delta$
\end{tabular}

A SUB instruction $l_{i}:\left(\operatorname{SUB}(r), l_{j}, l_{k}\right)$ (with $\left.r \in\{1,2\}\right)$ is simulated according to the four steps suggested in the table given above:

In the first step, we create a membrane with the label $r+1$, where $l_{i}$ is sent to, and simultaneously $d$ becomes $d^{\prime}$. In the next step, if any $a_{r}$ exists, i.e., if register $r$ is not empty, then one copy of $a_{r}$ should enter the membrane $r+1$ just having been created in the preceding step. Note that the selection of the 
right membrane (the use of $i n_{r+1}$ instead of $i n$ ) is important: $a_{r}$ has to go to the membrane created in the previous step, when $r+1$ has been specified by the label $l_{i}$. At the same time, $d^{\prime}$ enters the membrane $r+1$, and $l_{i}$ becomes $l_{i}^{\prime}$ in this membrane. If the register $r$ is empty, then the catalyst is doing nothing in this second step.

In the third step, in membrane $r+1, l_{i}^{\prime}$ becomes $l_{i}^{\prime \prime}$ and $d^{\prime}$ becomes $d^{\prime \prime}$. If $a_{r}$ is not present in membrane $r+1$, nothing else happens there in this step; if $a_{r}$ is present, it dissolves the membrane and disappears. Observe that in both cases $c a_{r} \rightarrow c\left(a_{r}, i n_{r+1}\right)$ will not be applicable (anymore) in $R_{1}$. Thus, we either have $c l_{i}^{\prime \prime} d^{\prime \prime}$ in the skin membrane (when the register has been non-empty), or we have only $c$ in the skin membrane and $l_{i}^{\prime \prime} d^{\prime \prime}$ in the inner membrane $r+1$. In the first case, in the fourth step we use the rules $c l_{i}^{\prime \prime} \rightarrow c l_{j}$ and $d^{\prime \prime} \rightarrow d$ from $R_{1}$, which is the correct continuation of the simulation of the SUB instruction; in the latter case, we use $l_{i}^{\prime \prime} \rightarrow l_{k}$ and $d^{\prime \prime} \rightarrow d \delta$ in $R_{r+1}$. The inner membrane is dissolved, and in the skin membrane we get the objects $c l_{k} d$. In both cases, the simulation of the SUB instruction is correct and we return to a configuration as that we started with, hence, the simulation of another instruction can start.

There is one interference between the rules of $\Pi$ simulating the ADD and the SUB instructions of $M$. If in the second step of simulating a SUB instruction, instead of $d^{\prime} \rightarrow\left(d^{\prime}, i n_{r+1}\right)$ we use $d^{\prime} \rightarrow d$, then the case when register $r$ is non-empty continues correctly, as the simulation lasts four steps, and in the end $d$ is present in the skin membrane (the dissolution of membrane $r+1$ is done by $a_{r}$ ). If the register $r$ has been empty, $l_{i}^{\prime \prime}$ will become $l_{k}$ in membrane $r+1$ and it will remain there until $d^{\prime}$ enters the membrane, changes to $d^{\prime \prime}$, and then dissolves it (as long as $d, d^{\prime}$ switch to each other in the skin membrane, the computation cannot halt). Thus, also in this case we have to return to the correct submultiset $c l_{k} d$ in the skin membrane.

Consequently, exactly the halting computations of $M$ are simulated by the halting computations in $\Pi$; hence, $P s(M)=P s(\Pi)$. The observation that the maximal number of membranes in any computation of $\Pi$ is two completes the proof.

It remains as an open problem whether it is possible to use the target in only instead of the $i n_{j}$.

\section{Computational Completeness of P Systems with Mobile Catalysts}

If the membrane creation rules are of the form $c a \rightarrow[c b]_{i}$, then this implicitly means that the catalyst is moving from one region to another one. However, for mobile catalysts, the computational completeness of such systems with only one catalyst has already been proved in [7], using three membranes and targets of the forms here, out, and $i n_{j}$. In this paper, we improve this result from the last point of view, making only use of the targets here, out, and in. In fact, if in the proof of Theorem 2 we let the catalyst $c$ move with all the other objects, then we immediately obtain a proof for $\mathrm{NOP}_{7}\left(\right.$ mcat $\left._{1}\right)=N R E$ where even only the targets out and in are used (but instead of three we need seven membranes).

Theorem $5 \mathrm{YOP}_{3}\left(\right.$ mcat $\left._{1}\right)=Y R E, Y \in\{N, P s\}$.

Proof. We only prove the inclusion $P s R E \subseteq P_{s} O_{3}\left(\right.$ mcat $\left._{1}\right)$. Let us consider a register machine $M=$ $\left(n+2, B, l_{0}, l_{h}, I\right)$ with only the first and the second register ever being decremented. Again we define $A=\left\{a_{1}, \ldots, a_{n+2}\right\}$ as the set of objects for representing the contents of the registers 1 to $n+2$ of $M$. We construct the following P system: 


$$
\begin{aligned}
\Pi= & \left(O,\{c\},\left[[]_{2}[]_{3}\right]_{1}, c l_{0}, R_{1}, R_{2}, R_{3}, 0\right), \\
O= & A \cup\left\{l, l^{\prime}, l^{\prime \prime}, l^{\prime \prime \prime} \mid l \in B\right\} \cup\{c, \#\}, \\
R_{1}= & \left\{l_{i} \rightarrow l_{j}\left(a_{r}, \text { in }\right), l_{i} \rightarrow l_{k}\left(a_{r}, \text { in }\right) \mid l_{i}:\left(\operatorname{ADD}(r), l_{j}, l_{k}\right) \in I, r \in\{1,2\}\right\} \\
\cup & \left\{l_{i} \rightarrow l_{j}\left(a_{r}, \text { out }\right), l_{i} \rightarrow l_{k}\left(a_{r}, \text { out }\right) \mid l_{i}:\left(\operatorname{ADD}(r), l_{j}, l_{k}\right) \in I, 3 \leq r \leq n+2\right\} \\
\cup & \left\{c l_{i} \rightarrow(c, \text { in })\left(l_{i}, \text { in }\right) \mid l_{i}:\left(\operatorname{SUB}(r), l_{j}, l_{k}\right) \in I, r \in\{1,2\}\right\} \\
\cup & \left\{c l_{i}^{\prime \prime \prime} \rightarrow c l_{j}, l_{i}^{\prime \prime \prime} \rightarrow \#, \# \rightarrow \# \mid l_{i}:\left(\operatorname{SUB}(r), l_{j}, l_{k}\right) \in I, r \in\{1,2\}\right\}, \\
R_{2}= & \left\{a_{2} \rightarrow \#, \# \rightarrow \#, c a_{1} \rightarrow(c, \text { out })\right\} \\
\cup & \left\{l_{i} \rightarrow \# \mid l_{i}:\left(\operatorname{SUB}(r), l_{j}, l_{k}\right) \in I, r \in\{1,2\}\right\} \\
\cup & \left\{c l_{i} \rightarrow c l_{i}^{\prime}, l_{i}^{\prime} \rightarrow l_{i}^{\prime \prime}, c l_{i}^{\prime \prime} \rightarrow(c, \text { out })\left(l_{k}, \text { out }\right), l_{i}^{\prime \prime} \rightarrow\left(l_{i}^{\prime \prime \prime}, \text { out }\right) \mid l_{i}:\left(\operatorname{SUB}(1), l_{j}, l_{k}\right) \in I\right\}, \\
R_{3}= & \left\{a_{1} \rightarrow \#, \# \rightarrow \#, c a_{2} \rightarrow(c, \text { out })\right\} \\
\cup & \left\{l_{i} \rightarrow \# \mid l_{i}:\left(\operatorname{SUB}(r), l_{j}, l_{k}\right) \in I, r \in\{1,2\}\right\} \\
\cup & \left\{c l_{i} \rightarrow c l_{i}^{\prime}, l_{i}^{\prime} \rightarrow l_{i}^{\prime \prime}, c l_{i}^{\prime \prime} \rightarrow(c, \text { out })\left(l_{k}, \text { out }\right), l_{i}^{\prime \prime} \rightarrow\left(l_{i}^{\prime \prime \prime}, \text { out }\right) \mid l_{i}:\left(\operatorname{SUB}(2), l_{j}, l_{k}\right) \in I\right\} .
\end{aligned}
$$

The rules in the sets of rules $R_{1}, R_{2}$, and $R_{3}$ are used as follows:

A. Let $l_{i}:\left(\operatorname{ADD}(r), l_{j}, l_{k}\right)$ be an ADD instruction in $I$. If $r \geq 3$, then the rules $l_{i} \rightarrow l_{j}\left(a_{r}\right.$, out $), l_{i} \rightarrow$ $l_{k}\left(a_{r}\right.$, out $)$ are used in $R_{1}$; if $r \in\{1,2\}$, in $R_{1}$ we take the rules $l_{i} \rightarrow l_{j}\left(a_{r}\right.$, in $)$ and $l_{i} \rightarrow l_{k}\left(a_{r}\right.$, in $)$ as well as the rules $a_{j} \rightarrow \#$ and \# $\rightarrow$ in $R_{4-j}, j \in\{1,2\}$. The contents of each register $r, r \in\{1,2\}$, is represented by the number of objects $a_{r}$ present in membrane $r+1$; any object $a_{r}, r \geq 3$, is immediately sent out into the environment. If $a_{j}$ is introduced in membrane $4-j, j \in\{1,2\}$, then the trap object \# is produced and the computation never halts.

B. The simulation of a SUB instruction $l_{i}:\left(\operatorname{SUB}(r), l_{j}, l_{k}\right)$ is carried out by the following rules (the simulation again has four steps, as in the proof of Theorem 4):

For the first step, we take the rule $c l_{i} \rightarrow(c, i n)\left(l_{i}, i n\right)$ in $R_{1}$ and the rule $l_{i} \rightarrow \#$ in both $R_{2}$ and $R_{3}$ (if $c$ and $l_{i}$ are not moved together into an inner membrane, then the trap object \# is produced and the computation never halts). In the second step, $R_{r+1}$ has to use the rule $c l_{i} \rightarrow c l_{i}^{\prime}$. This checks whether $c$ and $l_{i}$ have been moved together into the right membrane $r+1$; if this is not the case, then the rule $c l_{i} \rightarrow c l_{i}^{\prime}$ is not available and the rule $l_{i} \rightarrow$ \# must be used, which causes the computation to never halt.

Thus, after the second step, we know whether both $c$ and $l_{i}\left(l_{i}^{\prime}\right)$ are in the right membrane $r+1$. The rules $c a_{r} \rightarrow(c$, out $)$ and $l_{i}^{\prime} \rightarrow l_{i}^{\prime \prime}$ in $R_{r+1}$ are used in order to perform the third step of the simulation. If there is any copy of $a_{r}$ in membrane $r+1$ (i.e., if register $r$ is not empty), then the catalyst exits, while also removing a copy of $a_{r}$. Simultaneously, $l_{i}^{\prime}$ becomes $l_{i}^{\prime \prime}$. Hence, if the register $r$ has been non-empty, we now have $c$ in the skin membrane and $l_{i}^{\prime \prime}$ in membrane $r+1$; if register $r$ has been empty, we have both $c$ and $l_{i}^{\prime \prime}$ in membrane $r+1$. We then use the rules $c l_{i}^{\prime \prime} \rightarrow(c$, out $)\left(l_{k}\right.$, out $)$ and $l_{i}^{\prime \prime} \rightarrow\left(l_{i}^{\prime \prime \prime}\right.$, out $)$ in $R_{r+1}$ as well as the rules $c l_{i}^{\prime \prime \prime} \rightarrow c l_{j}$ and $l_{i}^{\prime \prime \prime} \rightarrow \#$ in $R_{1}$. If $c$ is inside membrane $r+1$, we get $c l_{k}$ in the skin membrane, which is the correct continuation for the case when the register is empty. If $c$ is not in membrane $r+1$, then $l_{i}^{\prime \prime}$ exits alone thereby becoming $l_{i}^{\prime \prime \prime}$, and, together with $c$, which waits in the skin membrane, introduces $l_{j}$, which is a correct continuation, too. If the rule $l_{i}^{\prime \prime} \rightarrow\left(l_{i}^{\prime \prime \prime}\right.$, out $)$ is used although $c$ is inside membrane $r+1$, then in the skin membrane we have to use the rule $l_{i}^{\prime \prime \prime} \rightarrow \#$ and the computation never halts (as we have the rule \# $\rightarrow$ \# in $R_{1}$ ).

In all cases, the simulation of the SUB instruction works correctly, and we return to a configuration with the catalyst and a label from $H$ in the skin region.

In sum, we have the equality $\operatorname{Ps}(M)=\operatorname{Ps}(\Pi)$, which completes the proof. 


\section{Final Remarks}

Although we have exhibited several new computational completeness results for P systems using only one catalyst together with some additional control mechanism, the original problem of characterizing the sets of (vectors of) non-negative integers generated by $\mathrm{P}$ systems with only one catalyst still remains open. A similar challenging problem is to consider purely catalytic $\mathrm{P}$ systems with only two catalysts: with only one catalyst, we obtain the regular sets; as shown in [4], three catalysts are enough to obtain computational completeness. With two catalysts and some additional control mechanism, computational completeness can be obtained, too, see [3].

Acknowledgements. The work of Gheorghe Păun has been supported by Proyecto de Excelencia con Investigador de Reconocida Valía, de la Junta de Andalucía, grant P08 - TIC 04200. The authors gratefully acknowledge the suggestions of the referees.

\section{References}

[1] A. Alhazov, R. Freund, H. Heikenwälder, M. Oswald, Yu. Rogozhin \& S. Verlan (2013): Sequential P systems with regular control. In E. Csuhaj-Varjú, M. Gheorghe, G. Rozenberg, A. Salomaa \& Gy. Vaszil, editors: Membrane Computing - 13th International Conference, CMC 2012, Budapest, Hungary, August 28-31, 2012, Revised Selected Papers, LNCS 7762, Springer, pp. 112-127, doi:10.1007/978-3-642-36751-9_9.

[2] J. Dassow \& Gh. Păun (1989): Regulated Rewriting in Formal Language Theory. EATCS Monographs in Theoretical Computer Science 18, Springer, doi $10.1007 / 978-3-642-74932-2$.

[3] R. Freund (2013): Purely catalytic P systems: two catalysts can be sufficient for computational completeness. In A. Alhazov, S. Cojocaru, M. Gheorghe \& Yu. Rogozhin, editors: CMC14 Proceedings - The 14th International Conference on Membrane Computing, Chişinău, August 20-23, 2013, Institute of Mathematics and Computer Science, Academy of Sciences of Moldova, pp. 153-166.

[4] R. Freund, L. Kari, M. Oswald \& P. Sosík (2005): Computationally universal P systems without priorities: two catalysts are sufficient. Theoretical Computer Science 330, pp. 251-266, doi $10.1016 / j$.tcs.2004.06.029.

[5] R. Freund \& Gh. Păun (2013): Universal P systems: one catalyst can be sufficient. In L. Valencia-Cabrera, M. García-Quismondo, L.F. Macas-Ramos, M.A. Martínez-del-Amor, Gh. Păun \& A. Riscos-Núñez, editors: Proceedings 11th Brainstorming Week on Membrane Computing, Sevilla, 4-8 February 2013, Fenix Editora, Sevilla, pp. 81-96.

[6] M.A. Gutiérrez-Naranjo \& M.J. Pérez-Jiménez (2013): P systems with membrane creation and rule input. To appear.

[7] S.N. Krishna \& A. Păun (2004): Results on catalytic and evolution-communication P systems. New Generation Computing 22, pp. 377-394, doi 10.1007/BF03037288,

[8] K. Krithivasan, Gh. Păun \& A. Ramanujan (2013): On controlled P systems. In L. Valencia-Cabrera, M. García-Quismondo, L.F. Macas-Ramos, M.A. Martínez-del-Amor, Gh. Păun \& A. Riscos-Núñez, editors: Proceedings 11th Brainstorming Week on Membrane Computing, Sevilla, 4-8 February 2013, Fenix Editora, Sevilla, pp. 137-151.

[9] K. Krithivasan M. Mutyam (2001): P systems with membrane creation: universality and efficiency. In M. Margenstern \& Yu. Rogozhin, editors: Proceedings MCU 2001, Lecture Notes in Computer Science 2055, Springer, pp. 276-287, doi 10.1007/3-540-45132-3_19.

[10] M.L. Minsky (1967): Computation: Finite and Infinite Machines. Prentice Hall, Englewood Cliffs, New Jersey.

[11] Gh. Păun (2000): Computing with membranes - a variant. International Journal of Foundations of Computer Science 11(1), pp. 167-182, doi $10.1142 / \mathrm{S} 0129054100000090$. 
[12] Gh. Păun (2003): Computing with membranes. Journal of Computer and System Sciences 61, pp. 108-143, doi: $10.1006 /$ jcss. 1999.1693

[13] Gh. Păun, G. Rozenberg \& A. Salomaa, editors (2010): The Oxford Handbook of Membrane Computing. Oxford University Press.

[14] G. Rozenberg \& A. Salomaa, editors (1997): Handbook of Formal Languages, 3 volumes. Springer.

[15] The P Systems Website: Available at http://ppage.psystems.eu. 